\title{
Differential expression of CD44v6 in metastases of intestinal and diffuse types of gastric carcinoma
}

\author{
E M Castellà, A Ariza, I Pellicer, A Fernández-Vasalo, I Ojanguren
}

\begin{abstract}
Aims-To assess whether standard and variant isoforms of CD44 (CD44s, CD44v5, and CD44v6) have a differential expression profile in early versus advanced gastric adenocarcinoma of the diffuse and intestinal types and their metastases.

Methods-Immunohistochemical expression of CD44s, CD44v5, and CD44v6 was evaluated in 14 early gastric cancers (nine intestinal and five diffuse) and 37 advanced adenocarcinomas ( 21 intestinal and 16 diffuse) as well as in 18 cases of perigastric lymph node metastasis. Ten normal and five metaplastic gastric mucosa samples were also included in the study.

Results-Although no significant association was found between the degree of invasion and the CD44 expression profile, CD44v6 positivity was detected more frequently in metastases of intestinal-type carcinomas $(66 \%)$ than in metastases of diffuse-type neoplasms $(11 \%)(p<0.05)$. Weak CD44s, CD44v5, and CD44v6 expression was observed focally in both normal and metaplastic gastric mucosa samples.

Conclusions-These data suggest that CD44v6 expression may be involved in the production of lymph node metastases in intestinal-type gastric carcinoma but not in the diffuse-type disease, the metastatic potential of which is most likely unrelated to the CD44 family of adhesion molecules. (f Clin Pathol 1998;51:134-137)
\end{abstract}

Dr Ariza.

email: a.ariza@cc.uab.es

Accepted for publication 11 November 1997

Keywords: gastric adenocarcinoma; intestinal carcinoma; diffuse carcinoma; CD44; immunohistochemistry; invasion; metastasis

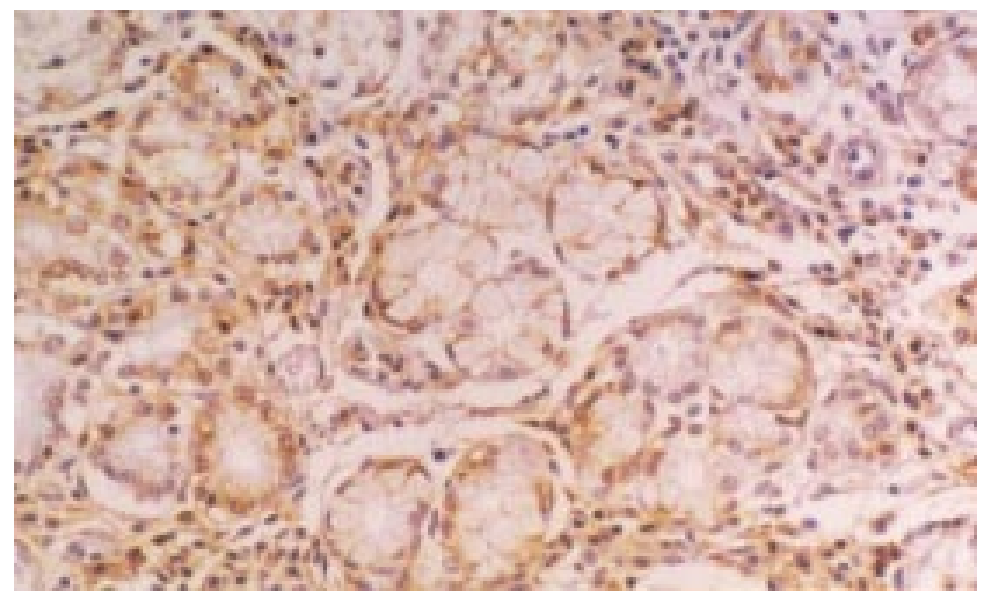

Figure 1 CD44s expression in normal antral gastric mucosa is detected in neck and isthmus mucous cells, as well as in lymphocytes.
The CD44 family of cell surface adhesion molecules is expressed in a variety of normal and neoplastic tissues. ${ }^{1}$ This protein family has been linked to a number of functions including lymphocyte homing and activation, ${ }^{23}$ haemopoiesis, cell-cell, and cell-extracellular matrix interactions, and cell migration. ${ }^{4}$ To be able to exert such diverse effects, CD44 generates numerous isoforms through a mechanism of alternative splicing. Some of these CD44 isoforms also seem to play a role in the production of tumour metastases. ${ }^{5}$ Specifically, a nonmetastatic cell line of a rat pancreatic adenocarcinoma has been shown to acquire metastatic potential when transfected with CD44 variants containing exon v6 (CD44v6), an affect that can be blocked by anti-v6 antibodies. $^{6-8}$

With regard to gastric adenocarcinoma, its intestinal and diffuse-types have been shown to express CD44 isoforms differentially. Thus, whereas intestinal-type adenocarcinomas express CD44 variant isoforms containing exons v5 and v6, diffuse-type tumours are associated predominantly with CD44 isoforms that include exon v5 (CD44v5). ${ }^{9}$ In addition, CD 44 seems to influence the prognosis of gastric carcinoma, because CD44 overexpression is associated with the development of an invasive phenotype, ${ }^{10}$ and high tumour recurrence and mortality rates in patients with curatively resected gastric cancer. ${ }^{11}$

In view of these relations between the biological behaviour of gastric carcinoma and its CD44 expression status, investigation into the possible differences between the CD44 expression profiles of early and advanced gastric cancers of intestinal and diffuse-types and their metastases would seem useful. Accordingly, we have evaluated immunohistochemically the expression of standard CD44 (CD44s), CD44v5, and CD44v6 in normal gastric mucosa, intestinal metaplasia of the stomach, and gastric carcinoma of the intestinal and diffuse types of various levels of invasion.

\section{Materials and methods}

Tissue blocks of normal and pathological stomach from 54 patients (31 men and 23 women) with a mean age of 67.4 years (range, 41-94 years) were obtained from the archives of the department of pathology, Hospital Germans Trias I Pujol. All patients underwent surgery between 1986 and 1995 at the same institution. The 84 samples studied were distributed as follows: five each of normal antral mucosa, normal fundic mucosa, and metaplastic mucosa obtained from gastrectomy specimens; 14 early gastric carcinomas (five of 


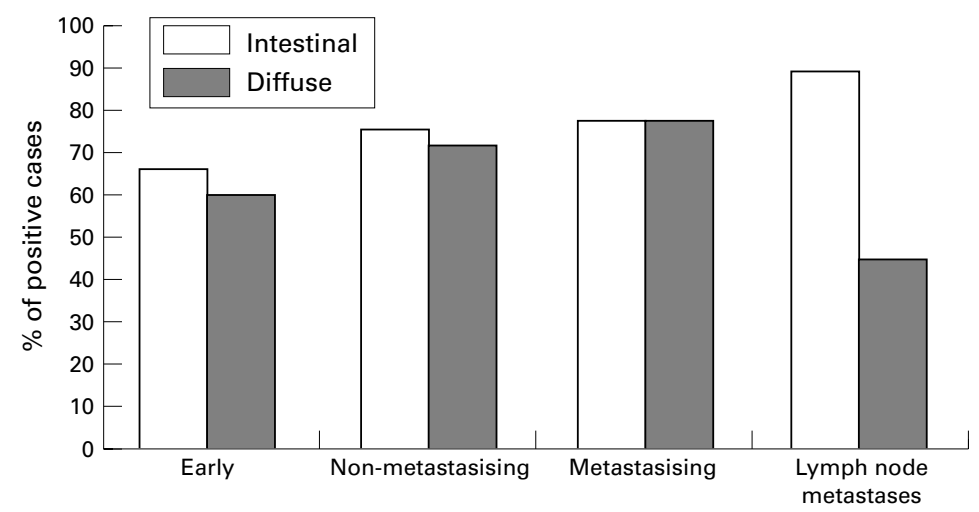

Figure 2 CD44s expression in intestinal and diffuse-type carcinoma of the stomach.

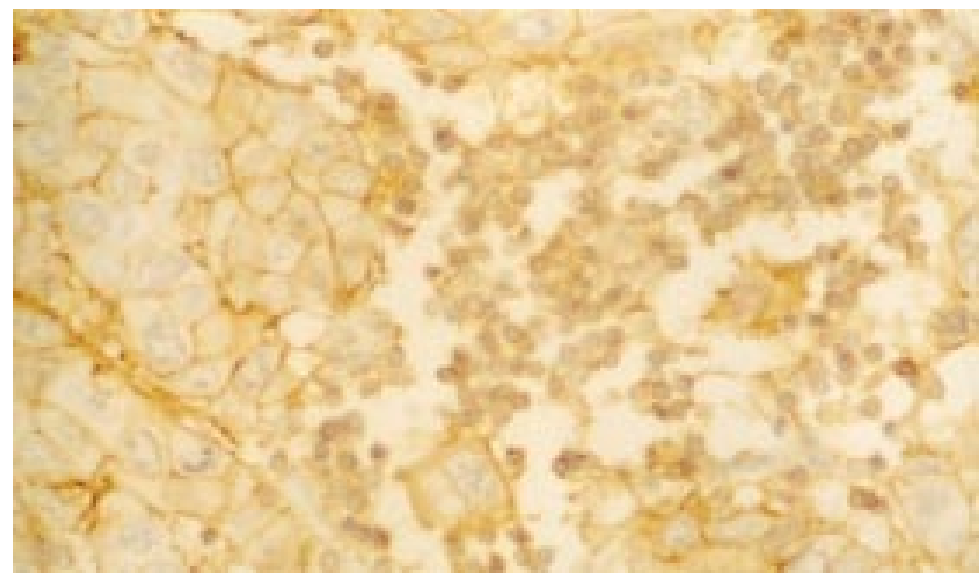

Figure 3 Intestinal-type adenocarcinoma with focal CD44v5 immunostaining in cell membranes.

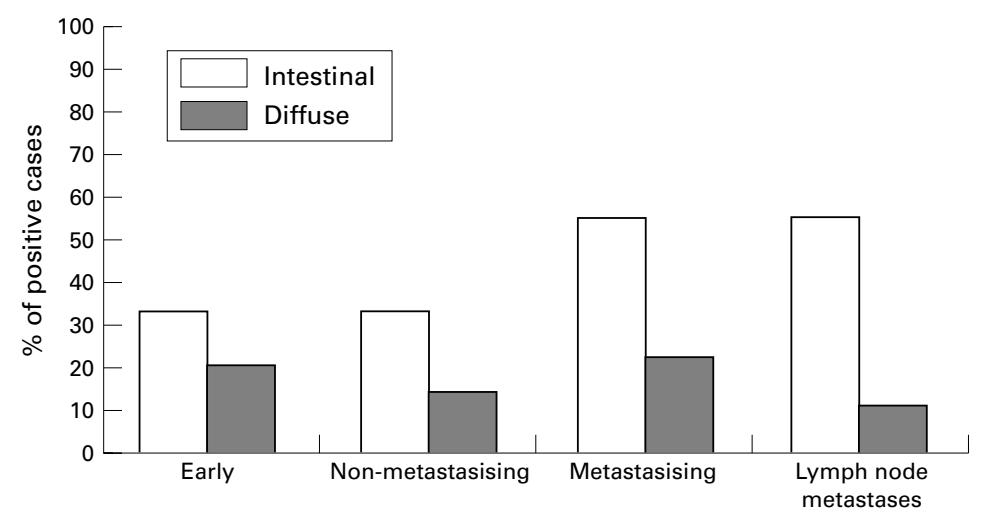

Figure 4 CD44v5 expression in intestinal and diffuse-type carcinoma of the stomach.

the diffuse-type and nine of the intestinal-type); and 37 advanced gastric carcinomas that invaded beyond the submucosa (21 of the intestinal-type (nine with lymph node metastasis), and 16 of the diffuse-type (nine with lymph node metastasis)). The degrees of differentiation of intestinal-type tumours were as follows: nine well differentiated, 11 moderately differentiated, and 10 poorly differentiated.

Histological features were evaluated according to internationally established criteria. The immunohistochemical results obtained from neoplastic samples were correlated with histological type and prognostic factors such as level of invasion, degree of differentiation, lymph vessel involvement, and evidence of metastasis at the time of diagnosis.
All biopsy specimens were fixed in $10 \%$ neutral formalin, embedded in paraffin wax at $57-60^{\circ} \mathrm{C}$, and stained with haematoxylin and eosin. For immunohistochemical studies, $5 \mu \mathrm{m}$ thick sections were deparaffinised and incubated with anti-CD44s (dilution, 1/1000; clone 2C5; RD Systems, Abingdon, UK), antiCD44v6 (dilution, 1/1000; clone 2F-1D; RD Systems), and anti-CD44v5 (dilution, 1/2500; clone VFF-8; Bender MedSystems, Vienna, Austria) mouse monoclonal antibodies for 22 hours at room temperature. Staining was visualised using the avidin-biotin-peroxidase complex (Dako, Glostrup, Denmark) technique. A non-immune mouse serum was used as a negative control instead of the specific monoclonal antibodies. A non-small cell lung carcinoma was used as a positive control for CD44s and CD44v6, and a colon adenocarcinoma was used as a positive control for CD44v5.

Two features were taken into account for the evaluation of CD44 expression: the percentage of positively stained tumour cells (negative, $<5 \%$; focal, between $5 \%$ and $50 \%$; diffuse, $>50 \%$ ), and the intensity of staining: 0 , no staining; 1, weak staining; 2 , moderate staining; and 3, strong staining (comparable with lymphocytes). Only cell membrane staining was considered to be positive. Two of the authors evaluated the slides independently. Cases with dissimilar observations were reviewed to reach a consensus.

The data were compared with the aid of Fisher's exact test to detect possible associations between CD44 expression and histological tumour type, level of tumour invasiveness, or lymph vessel involvement. $\mathrm{p}$ values under 0.05 were regarded as statistically significant.

\section{Results}

CD44 IMMUNOREACTIVITY IN NORMAL AND METAPLASTIC GASTRIC MUCOSA

Ten samples of normal antral or fundic mucosa and five samples of intestinal metaplasia of the stomach were tested for immunoreactivity with anti-CD44s, anti-CD44v5, and anti-CD44v6 monoclonal antibodies. CD44s was expressed in most samples examined, specifically in neck and isthmus mucous cells (fig 1), as well as in metaplastic areas.

Membrane positivity for v5 and v6 was present preferentially in the same proliferative and metaplastic areas, although the immunostaining was weaker and more focal in distribution. CD 44s and isoforms containing exons v5 and $\mathrm{v} 6$ were not expressed in the foveolar epithelium or the luminal surface.

\section{CD44 IMMUNOREACTIVITY IN GASTRIC}

\section{CARCINOMAS}

Intestinal-type adenocarcinomas

Early intestinal adenocarcinoma showed CD44s immunopositivity in $66 \%$ of cases. Advanced adenocarcinomas of the intestinal type were CD44s positive in $75 \%$ of nonmetastasising cases and in $77 \%$ of metastasising tumours (fig 2).

CD44v5 was expressed in 33\% of early and advanced non-metastasising carcinomas, whereas $55 \%$ of metastasising cases were 


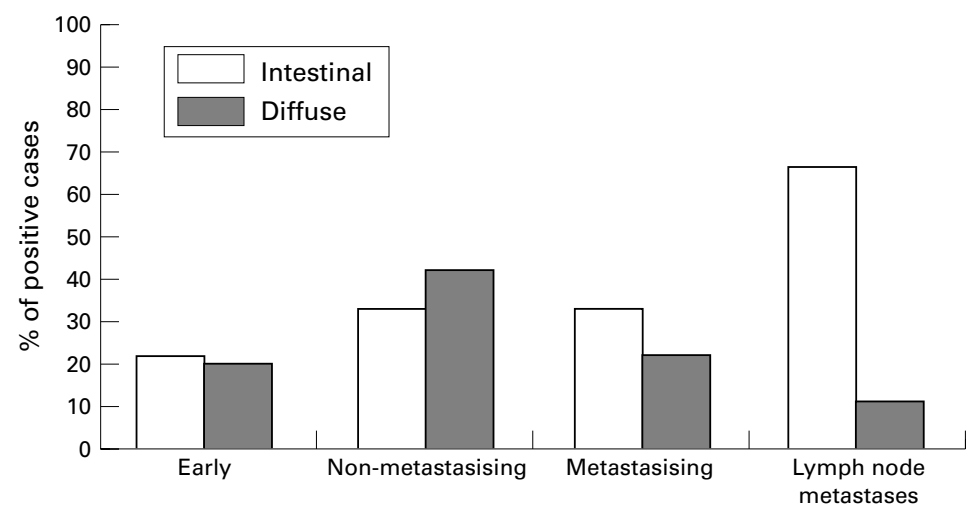

Figure 5 CD44v6 expression in intestinal and diffue-type carcinoma of the stomach.

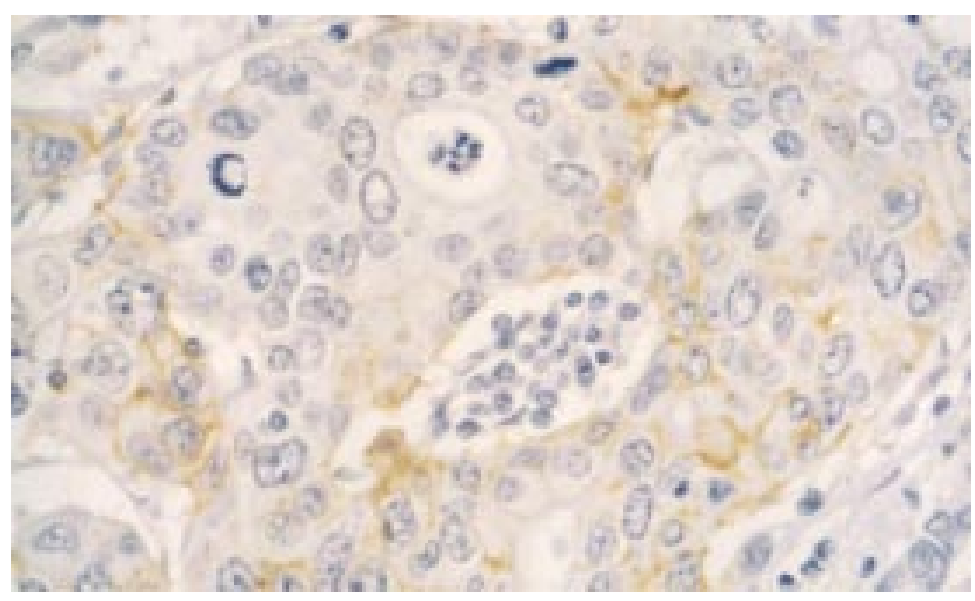

Figure 6 Lymph node metastasis of intestinal-type gastric adenocarcinoma: diffuse and intense CD44s immunoreactivity in lymphocytes and epithelial cells.

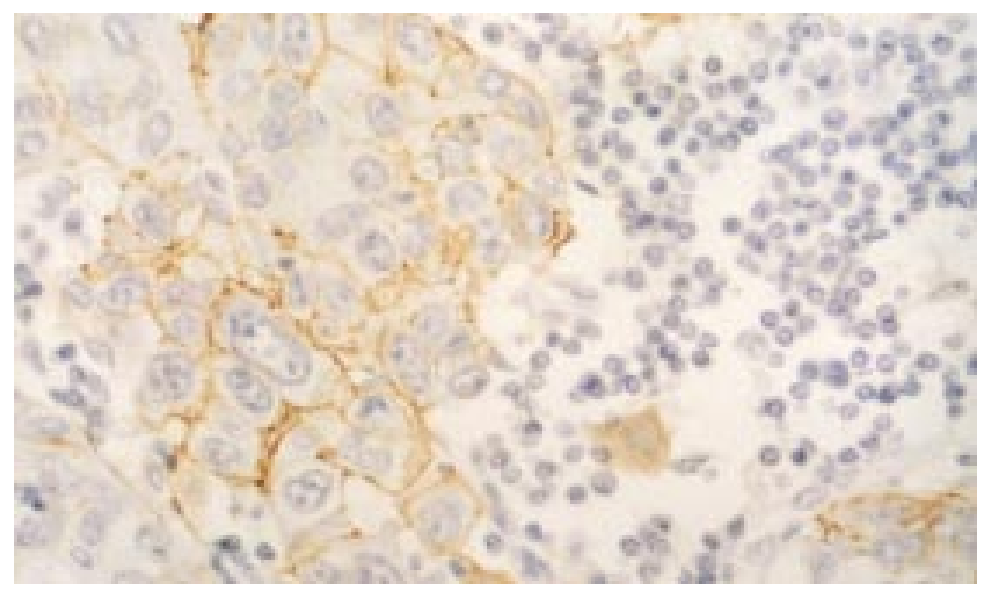

Figure 7 Lymph node metastasis of intestinal-type gastric adenocarcinoma: focal CD44v6 immunostaining restricted to epithelial cells.

immunoreactive for this antibody (fig 3) $(\mathrm{p}=0.2)$ (fig 4).

Positive immunostaining with CD44v6 was obtained in $22 \%$ of early intestinal adenocarcinoma samples. As for advanced intestinal cases, non-metastasising and metastasising tumours expressed CD44v6 positivity with an identical frequency (33\%) (fig 5).

Diffuse-type adenocarcinomas

Sixty per cent of early diffuse adenocarcinomas showed CD44s immunostaining. Nonmetastasising and metastasising advanced cases were CD44 positive with a similar frequency ( $71 \%$ and $77 \%$, respectively). The difference between early and advanced diffuse adenocarcinomas did not reach statistical significance $(\mathrm{p}=0.5)$ (fig 2$)$.

CD44v5 expression rates of early (20\%) and advanced cases (14\% for non-metastasising and $22 \%$ for metastasising instances) of diffuse adenocarcinomas were quite similar (fig 4).

As for CD44v6, non-metastasising advanced carcinomas showed immunoreactivity more commonly $(42 \%)$ than early carcinomas $(22 \%)$, although the difference did not reach statistical significance $(p=0.4)$. Only $22 \%$ of metastasising cases were positive for CD44v6 (fig 5).

\section{LYMPH NODE METASTASES}

Regarding lymph node metastases, CD44s was expressed in a higher percentage of intestinal carcinomas (88\%) (fig 6) than diffuse-type cases $(44 \%)(p=0.06)$ (fig 2$)$.

CD44v5 expression was also detected more frequently in metastases of intestinal carcinomas $(55 \%)$ than in metastases of diffuse-type tumours $(11 \%)(\mathrm{p}=0.06)$ (fig 4$)$.

The CD44v6 expression rate in intestinaltype metastases (66\%) (fig 7) was higher than in diffuse-type metastases (11\%), the difference being statistically significant $(p=0.02)$ (fig 5).

The differentiation grade was not related to CD44s, CD44v5, or CD44v6 expression (data not shown). The intensity and distribution of immunostaining did not correlate with the level of invasion or the histological type of the carcinoma.

\section{Lymph vessel involvement}

We evaluated the possible correlation between CD44 positive metastases and lymph vessel involvement with the aid of Fisher's exact test. We did not find an association of standard or variant isoform expression and lymph vessel invasion.

The percentage of cases with CD44 positive tumour cells within lymph vessels is as follows: intestinal metastasising carcinoma expresses immunoreactivity in $80 \%$ of cases for CD $44 \mathrm{~s}$, in $17 \%$ for CD $44 v 5$, and in $33 \%$ for CD $44 v 6$. For diffuse-type gastric cancer these percentages are $75 \%, 12 \%$, and $25 \%$, respectively. These expression rates do not differ greatly from the results obtained for tumour cells not within lymph vessels.

\section{Discussion}

In this study, normal gastric mucosa showed focal immunostaining for CD44s. The epithelial cells expressing the highest levels of the standard isoform are the mucinous cells at the neck and isthmus of the antral or fundic glands. These areas correspond to the regenerative region of the gastric mucosa and seem to exhibit a pattern of CD44s expression which is quite similar to that shown by colonic basal cells. ${ }^{12}$

The two variant isoforms studied (CD44v5 and CD44v6) showed focal immunostaining in mucous cells of the antral and fundic mucosa and in metaplastic areas. In previous studies, 
Heider et al found v5 expression in the foveolar proliferation zone and mucous surface epithelium, whereas intestinal metaplasia reacted with specific monoclonal antibodies for exons v5 and v6. ${ }^{9}$

In the carcinoma samples investigated, CD44s immunopositivity seemed to increase with the depth of invasion, the percentage of positive cases being greater in advanced than in early carcinomas, although statistically significant differences could not be detected. Consequently, with respect to standard isoform expression, our results are consistent with those of Mayer et al, ${ }^{11}$ who found that CD $44 \mathrm{~s}$ and CD44v9 expression is associated with the presence of distant metastases at the time of diagnosis and with increased local recurrence and mortality rates in patients with curatively resected tumours.

The expression of variant isoforms containing spliced exon v6 has been associated previously with an unfavourable outcome in non-Hodgkin's lymphoma. ${ }^{13}$ Regarding epithelial tumours, Kaufmann et al demonstrated that CD44v6 is a good prognostic marker in breast carcinoma. ${ }^{14}$ On the other hand, expression of CD44 variant isoforms containing v6 exon has been seen to increase in parallel with colorectal tumour progression and Dukes' stage. ${ }^{12}$ However, other authors ${ }^{15}$ have claimed that CD44v6 expression has a prognostic value which is independent of Dukes' stage.

Previous studies of gastric cancer have reported that intestinal-type adenocarcinoma expresses variant isoforms containing exons v5 and v6, whereas diffuse-type adenocarcinoma expresses predominantly exon $\mathrm{v} 5$ and, in a very low percentage of cases, exon v6. In addition, the lymph node metastases of these diffusetype tumours have been shown to be devoid of v6 related proteins by Southern blot analysis. In contrast, a correlation between primary tumours of intestinal type and their lymph node metastases has been observed in regard to v6 containing isoforms, while no obvious correlation was detected for exon v5. ${ }^{9}$ More recently, Dammrich et al claimed that the relation between v6 and metastatic lymphogenic spreading is different in the two cancer types. ${ }^{16}$ Specifically, whereas diffuse-type metastasising cells lack v6 expression, intestinal-type cancers do show v6 staining in infiltrative lymph node metastases.

Our results are in agreement with these previous observations. In our hands, isoforms containing exon v6 were detected more frequently in metastasising intestinal-type carcinomas than in metastasising diffuse-type carcinomas. Moreover, CD44v6 expression was significantly more frequent in lymph node metastases of intestinal-type carcinomas than in those of diffuse-type cases. These findings suggest an important role for exon v6 in the metastatic process, particularly in the lymph node spreading of intestinal adenocarcinoma cells. On the contrary, invasion of lymph nodes by diffuse-type tumour cells seems to be independent of exon v6.

As for the level of invasion, we have not found statistically significant differences in standard and variant CD44 isoform expression between early and advanced cases of both histological types of gastric adenocarcinoma. These results may indicate that CD44 participation in the metastatic cascade takes place at the initial stages of tumour progression, because expression of CD44 variant isoforms is already detected in early carcinomas of both the intestinal and diffuse types. Further studies are necessary to elucidate the mechanisms that drive neoplastic cell invasion and migration to lymph nodes, as well as the degree of participation of CD44 in this process.

This work was supported by Spain's Fondo de Investigación Sanitaria (Grant No. 95/1345)

1 Fox SB, Fawcett J, Jackson DG, et al. Normal human issues, in addition to some tumors, express multiple different CD44 isoforms. Cancer Res 1994;54:4539-46.

2 Picker LJ, Nackache M, Butcher EC. Monoclonal antibodies to human lymphocyte homing receptors define a novel class of adhesion molecules on diverse cell types. 7 Cell Biol 1989;109:927-37.

3 Denning SM, Le PT, Singer KH, et al. Antibodies against the CD44 p80, lymphocyte homing receptor augment human peripheral blood $T$ cell activation. F Immunol 1990; 144:7-15.

4 Lesley J, Hyman R, Kinkade PW. CD44 and its interaction with the extracellular matrix. Adv Immunol 1993;54:271335

5 Herrlich P, Zoller M, Pals ST, et al. CD44 splice variants: metastases meet lymphocytes. Immunol Today 1993;14: 395-9.

6 Günthert U, Hofmann M, Wolfgang R, et al. A new variant of glycoprotein CD44 confers metastatic potential to rat carcinoma cells. Cell 1991;65:13-24.

7 Hofmann M, Rudy W, Zöller M, et al. CD44 splice variants confer metastatic behavior in rats: homologous sequences are expressed in human tumor cell lines. Cancer Res 1991; 51:5292-7.

8 Seiter S, Arch R, Reber S, et al. Prevention of tumor metastasis formation by antivariant CD44. F Exp Med 1993;177: 433-55.

9 Heider KH, Dämmrich J, Skroh-Angel P, et al. Differential expression of CD44 splice variants in intestinal and expression of CD44 splice variants in intestinal and diffuse-type human gastric carcinomas

10 Washington K, Gottfried MR, Telen MJ. Expression of the cell adhesion molecule CD44 in gastric adenocarcinoma. Hum Pathol 1994;25:1043-9.

11 Mayer B, Jauch KW, Günthert U, et al. De-novo expression of CD44 and survival in gastric cancer. Lancet 1993;342: 1019-22.

12 Wielenga VJM, Heider KH, Offerhaus GJA, et al. Expression of CD44 variant proteins in human colorectal cancer is related to tumor progression. Cancer Res 1993;53:47546.

13 Ristamäki R, Joensuu H, Söderström K, et al. CD44v6 expression in non-Hodgkin lymphoma: an association with low histological grade and poor prognosis. $f$ Pathol 1995;176:259-67.

14 Kaufmann M, Heider KJ, Sinn HP, et al. CD44 variant exon epitopes in primary breast cancer and length of survival. epitopes in primary brea
Lancet 1995;345:615-19.

15 Mulder JW, Kruyt PM, Sewnath M, et al. Colorectal cancer prognosis and expression of v6-containing CD44 proteins. Lancet 1994;334:1470-2.

16 Dammrich J, Vollmers HP, Heider KH, et al. Importance of different CD44v6 expression in human gastric intestinal and diffuse type cancers for metastatic lymphogenic spreading. f Mol Med 1995;73:395-401. 